\title{
Negative Thinking by Incremental Problem Solving: Application to Unate Covering
}

\author{
Evguenii I. Goldberg $\dagger^{\dagger} \ddagger \quad$ Luca P. Carloni ${ }^{\ddagger} \quad$ Tiziano Villa ${ }^{\S} \quad$ Robert K. Brayton ${ }^{\ddagger}$ \\ Alberto L. Sangiovanni-Vincentelli $\ddagger$ \\ $\ddagger$ Department of EECS \\ $\dagger$ Academy of Sciences of \\ $\S$ PARADES, \\ University of California at \\ Berkeley,Berkeley, CA 94720 \\ Belarus, Minsk \\ Via di S.Pantaleo, \\ 66, 00186 Roma
}

\begin{abstract}
We introduce a new technique to solve exactly a discrete optimization problem, based on the paradigm of "negative" thinking. The motivation is that when searching the space of solutions, often a good solution is reached quickly and then improved only a few times before the optimum is found: hence most of the solution space is explored to certify optimality, but it does not yield any improvement of the cost function. So it is quite natural for an algorithm to be "skeptical" about the chance to improve the current best solution.

For illustration we have applied our approach to the unate covering problem. We designed a procedure, raiser, implementing a negative thinking search, which is incorporated into a common branch-and-bound procedure. Raiser is invoked at a node of the search tree which is deep enough to justify negative thinking.

Raiser tries to detect a hard core of the matrix corresponding to the node by augmenting an independent set of rows in order to increase incrementally the cost of the minimum solutions covering the matrix. Eventually either raiser prunes the subtree rooted at the node (having found a lower bound equal or greater than the current best solution) or returns a new solution that becomes the current best one.

Experiments show that our program, AURA, outperforms both ESPRESSO and our enhancement of ESPRESSO using Coudert's limit lower bound [3]. It is always faster and in the most difficult examples either has a running time better by up to two orders of magnitude, or the other programs fail to finish due to timeout or spaceout. The package SCHERZO is faster on some examples and loses on others, due to a less powerful pruning strategy of the search space, partially mitigated by a more effective computation of the maximal independent set.
\end{abstract}

\section{Introduction}

A common approach to find an exact solution to problems in combinatorial optimization is branch-and-bound (BAB), which improves over exhaustive enumeration, because it avoids the exploration of some regions of the solution space, when it can certify by means of lower bounds that they do not contain a solution better than the current best one.

To ground the exposition in a concrete domain, in this paper we consider BAB applied to the solution of the Unate Covering Problem (UCP), that is of great interest in logic synthesis and operations research [4]. For the sake of simplicity we consider the case of UCP where all columns have the same cost. Such version of UCP is defined as follows. Given a Boolean matrix $A$ (all entries are $O$ or 1), find a minimum size subset of columns of $A$ such that every row of $A$ is covered by at least one column of the subset. A row $i$ is covered by a column $j$ if $A_{i j}=1$. We will denote an instance of
$\mathrm{UCP}$ with matrix $A$ as $U C P(A)$.

An exact solution of UCP is typically obtained by a branch-andbound recursive algorithm, which has been implemented in successful computer programs [7, 6]. Branching is done by columns, i.e., subproblems are generated by considering whether a chosen branching column is or is not in the solution.

A run of the algorithm, call it mincov, can be described by its computation tree. The root of the computation tree is the input of the problem, an edge represents a call to mincov, an internal node is a reduced input. A leaf is reached when a complete solution is found or the search is bounded away. From the root to any internal node there is a unique path, which is the current path for that node. The path leading to the node gives a partial solution and a submatrix $A_{N}$ obtained from $A$ by removing some rows and columns. On the path some columns are included in the partial solution; we denote by $\operatorname{path}\left(A_{N}\right)$ the set of columns included in the partial solution.

Suppose that we know that any minimal cover of $A_{N}$ is greater or equal to a value $L\left(A_{N}\right)$. The value is called a lower bound of the solutions of $U C P\left(A_{N}\right)$. So the size of any solution of $U C P(A)$ including the columns in path $\left(A_{N}\right)$ is greater or equal to $L\left(A_{N}\right)+$ $\left|p a t h\left(A_{N}\right)\right|$. So if we found before a solution best with the same or a smaller number of columns, i.e., $\mid$ best $\mid \leq L\left(A_{N}\right)+$ path $\left(A_{N}\right)$ we can stop the recursion and backtrack to the parent node of $A_{N}$.

Denote by $K\left(A_{N}\right)$ the value $\mid$ best $\left|-L\left(A_{N}\right)-\right| \operatorname{path}\left(A_{N}\right) \mid$. The condition to stop the recursion is given by $K\left(A_{N}\right) \leq 0$. On the other hand, if $K\left(A_{N}\right)$ has a large positive value, usually it means that $L\left(A_{N}\right)$ is far from the size of a minimal solution to $U C P\left(A_{N}\right)$ and so a lot of branching is expected from $A_{N}$ before a leaf can be reached.

Suppose that there is no way of improving the solution best in the search tree rooted at $A_{N}$, yet $K\left(A_{N}\right)$ is positive. Usually a branch-and-bound algorithm must continue branching. However, there is another way of making $K\left(A_{N}\right)$ negative or zero: it is to improve the lower bound $L\left(A_{N}\right)$.

The first way is "positive", in the sense that the algorithm tries to construct a better solution, and branching columns are chosen in the hope of improving the current best solution. The second way is "negative", in the sense that the algorithm tries to disprove that there is a better solution in the tree rooted at $A_{N}$.

To compare the role of "negative" and "positive" ways of search, notice that at the $n$-th level of the computation tree we can have up to $2^{n}$ nodes, i.e., subproblems. It is an experimental fact that usually in the first leaf a solution very close to the minimum one is found, so only few improvements are required to get a minimum solution. Therefore "positive" search will succeed and yield a new better solution only in a few of the $2^{n}$ subproblems. In the overwhelming majority of the subproblems "negative" search is more natural. The less frequently the best current solution is improved during the search, the more "negative" search is justified. In tum this is 
related to how much the solution space is "diversified", i.e., different solutions have different costs. Notice that BAB uses "negative thinking" in optimization problems by finding lower bounds, and in decision problems by checking the consistency of the partial solution with the current subproblem.

To exploit both "positive" and "negative" search, BAB is modified as follows. We start solving the initial problem with "positive thinking" in the ordinary column branching mode, called PTmode. Then, when the number of subproblems generated in the column branching mode becomes large "enough", each subproblem is solved in the "negative thinking" mode, called NT-mode. In optimization problems modes are switched depending on the ratio of the expected number of improvements to the number of subproblems generated at this level of the search tree. The smaller the ratio, the more appropriate is to switch to the NT-mode.

Let $P$ be a subproblem to be solved in NT-mode and suppose that if the cost of $P$ is greater than a given ubound then solving $P$ cannot give a better solution (w.l.o.g., assume to solve a minimization problem). The aim of the algorithm in the NT-mode is to prove that there is no solution of $P$ with cost less than ubound.

We propose a new way to implement "negative thinking": incremental problem solving (IPS). When solving a problem $A$ incrementally, we start with a subproblem $A^{\prime}$ of $A$, such that the solutions of $A^{\prime}$ can be represented compactly. Then we modify gradually $A^{\prime}$ by making it more complex to come closer to the full problem $A$ and we recompute the set of solutions of the modified problem. When applying "negative thinking", we try to find first the most difficult "obstacles" in the sequence from $A^{\prime}$ to $A$ with the goal to prove that no solution of $A^{\prime}$ can overcome the obstacles and be extended to a solution of $A$.

More precisely, let $P^{\prime}$ be a subproblem of $P$ such that its set of solutions $\operatorname{Sol}\left(P^{\prime}\right)$ can be represented in a compact form. Each solution of $P^{\prime}$ from $S o l\left(P^{\prime}\right)$ can be considered as a seed from which one may grow some solutions of $P$. In the NT-mode, the algorithm tries to show that no solution of $P$ with $\operatorname{cost}(P)<u b o u n d$ can grow from any solution $S \in \operatorname{Sol}\left(P^{\prime}\right)$. A naive approach is to form a sequence of problems $P_{1}, \cdots, P_{n}$, where $P_{1}=P^{\prime}$ and $P_{n}=P$. At each step one recomputes $\operatorname{Sol}\left(P_{i}\right)$ starting from $\operatorname{Sol}\left(P_{i-1}\right)$ and discards all solutions in $\operatorname{Sol}\left(P_{i}\right)$ with a cost greater than ubound. If, after removing the solutions costing more or as ubound, $\operatorname{Sol}(P i)=\emptyset$, for some $P_{a}, i \leq n$, then there is no solution of $P$ with cost less than ubound. A direct implementation of this approach has two drawbacks:

1. The size of the representation of $\operatorname{Sol}\left(P_{i}\right)$ may grow exponentially.

2. There are different ways of approaching $P$ from $P^{\prime}$. Each specific seed solution $S \in S o l\left(P^{\prime}\right)$ is extended more quickly to a solution costing more or as ubound by a specific sequence of augmentations, different from those appropriate for another solution $\hat{S} \in \operatorname{Sol}\left(P^{\prime}\right)$.

As a remedy we propose the paradigm of clusterization of solutions. We group in a cluster the solutions that are similar, in the sense of having the same witnesses of the fact that they cannot produce solutions of $P$ costing less than ubound.

In this paper we present an incremental UCP solver called raiser. Although we demonstrate our technique on UCP it can be applied to any discrete optimization problems with a monotone cost function, i.e., for which a minimum solution of a subproblem has a smaller cost than that of the initial problem.

The paper is organized as follows. Section 2 shows how an incremental solver is incorporated into the standard branch-andbound procedure for UCP. The idea of incremental improvement of the lower bound is sketched in Section 3, while Section 4 describes how the solutions of UCP are represented and recomputed. The raising procedure is explained in detail in Section 5 and experimental results are discussed in Section 6 . Conclusions are given in Section 7

\section{Incorporating an Incremental Solver into Branch- and-Bound}

The flow of a UCP solver based on branch-and-bound is shown in Fig. 1. The parts of text in bold font refer to the incremental solver and will be explained below. For details the reader is referred to [4]. Given a matrix $A$, existing $U C P$ solvers employ column branching to decompose the problem and use a maximal set of independent (non-intersecting) rows (MSIR) to compute a lower bound of $U C P(A)$ (since no column covers two rows from $M S I R)^{1}$.

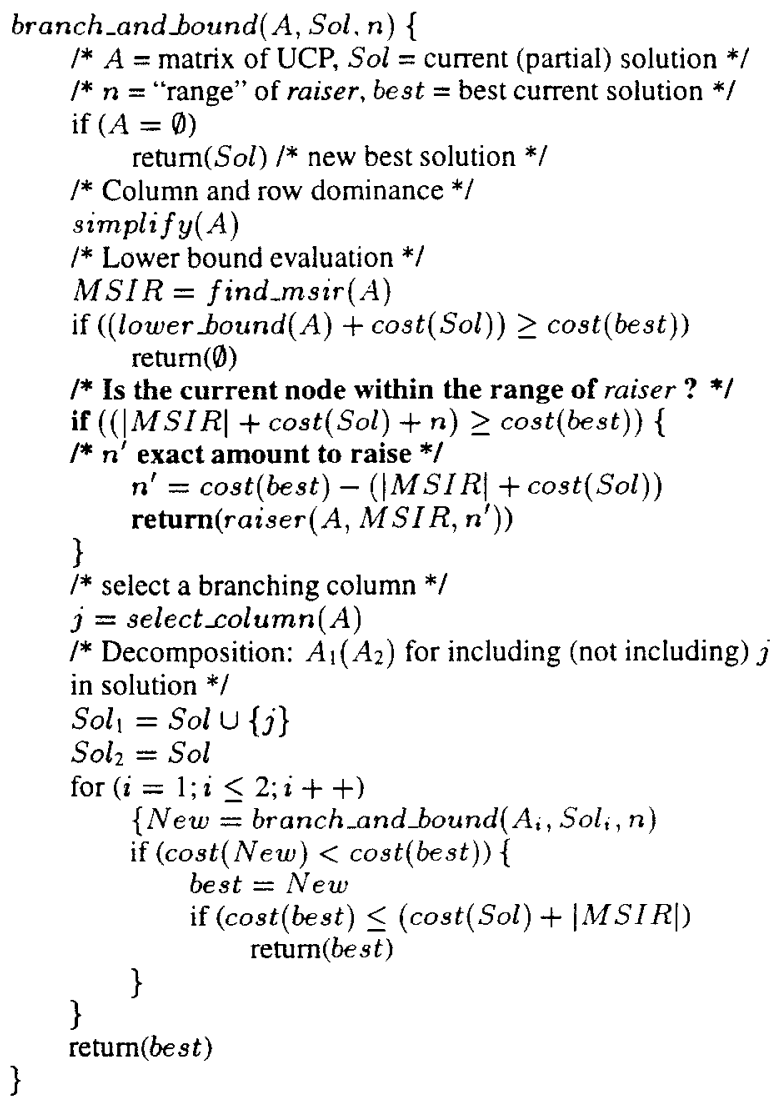

Figure 1: Branch-and-Bound enhanced by incremental solver

A procedure $n$-raiser, performing "negative thinking", is invoked when $M S I R$ is a lower bound not sufficient to prune the subtree rooted at the current node, but increasing the lower bound by $n$ would allow such pruning. The $n$-raiser starts from the subproblem $U C P(M S I R)$ whose solution space is very regular and then tries to extend it gradually to $A$. The $n$-raiser either returns a minimum cost solution of $U C P(A)$, if the lower bound cannot be raised by $n$, or retums the empty solution.

${ }^{1} \mathrm{~A}$ lower bounding technique based on linear programming relaxation, as commonly done in ILP, has been tested successfully for solving covering problems and reported in [5]. 
The parameter $n$ is specified a-priori and is the same for all invocations of raiser in the column branching mode. The value of $n$ is usually a small number in the range from 2 to 4 for two reasons:

1. if $n$ is small then the node is deep enough to warrant the application of negative thinking,

2. if $\boldsymbol{n}$ is small then one can make use of the fact that $U C P(M S I R)$ has a regular solution space.

Note that improving the lower bound even by a small amount may lead to considerable runtime reductions. For example, in [1] it was reported a new technique for pruning the search tree called limit lower bound. Sometimes the technique allows to reduce the search tree size by ten times. It can be shown that the limit lower bound technique prunes no more branches of the search tree than 1-raiser. and 5 .

The detailed description of the raiser is given in Sections 3, 4

\section{Incremental Improvement of the Lower Bound}

Given an optimization problem such that for any subproblem the cost of a minimum solution of the problem is greater than or equal to that of the subproblem, the size of a minimum solution of the subproblem gives a lower bound on the the size of a minimum solution of the problem (called cost monotonicity assumption). This fact is of practical interest if it is not difficult to find a minimum solution of the subproblem.

Denote by $\min (U C P(A))$ the size of a minimum solution of $U C P(A)$ and let $A^{\prime}$ be a submatrix of matrix $A$, consisting of some rows of $A$, i.e., $\operatorname{Col}(A)=\operatorname{Col}\left(A^{\prime}\right)$ and $\operatorname{Row}\left(A^{\prime}\right) \subseteq \operatorname{Row}(A)$. Any $U C P\left(A^{\prime}\right)$ where $A^{\prime}$ is a submatrix of $A$ satisfies the cost monotonicity assumption, since $\min \left(U C P\left(A^{\prime}\right)\right) \leq \min (U C P(A))$. We call lower bound submatrix a submatrix $\bar{A}^{\prime}$ whose minimum solution is used for evaluating a lower bound for $U C P(A)$. If $A^{\prime}$ is a $M S I R$ then $\min \left(U C P\left(A^{\prime}\right)\right)=\left|\operatorname{Row}\left(A^{\prime}\right)\right|$. We are now going to describe the idea underlying the method for an incremental improvement of the lower bound.

Denote by $A^{\prime}+A_{r}$ the submatrix of $A$ obtained by adding to $A^{\prime}$ a row $A_{r} \in \operatorname{Row}(A) \backslash \operatorname{Row}\left(A^{\prime}\right)$. Let $S$ be a solution of $U C P(A)$. A column $j \in S$ is called redundant if $S \backslash\{j\}$ is also a solution of $U C P(A)$. If a solution of $U C P(A)$ does not contain redundant columns then it is said to be irredundant. Denote by $\operatorname{Sol}\left(A^{\prime}, m\right)$ the set of solutions of $U C P\left(A^{\prime}\right)$ which includes all the irredundant solutions consisting of $m$ or fewer columns. So $\operatorname{Sol}\left(A^{\prime}, m\right)$ contains all the irredundant solutions of size from $\min \left(U C P\left(A^{\prime}\right)\right)$ to $m$ columns. So if $m=\min \left(U C P\left(A^{\prime}\right)\right)$ then $\operatorname{Sol}\left(A^{\prime}, m\right)$ gives exactly the set of all minimum solutions of $U C P\left(A^{\prime}\right)$.

Suppose that for a lower bound submatrix $A^{\prime}$ of $A$ we know a set of solutions $\operatorname{Sol}\left(A^{\prime}, m\right)$. The lower bound given by $A^{\prime}$ is equal to $m=\min \left(U C P\left(A^{\prime}\right)\right)$. Let us add a row $A_{p}$ of $A$ to $A^{\prime}$. Obviously $\operatorname{Sol}\left(A^{\prime}+A_{p}, m\right) \subseteq \operatorname{Sol}\left(A^{\prime}, m\right)$, since in general some solutions from $\operatorname{Sol}\left(A^{\prime}, m\right)$ do not cover $A_{p}$ and so are not contained in $\operatorname{Sol}\left(A^{\prime}+A_{p}, m\right)$. So after having added a set of rows $A_{i_{1}}, . ., A_{i_{k}}$ of $A$ to $A^{\prime}$, we can reach a stage when $\operatorname{Sol}\left(A^{\prime}+A_{i_{1}}+. .+A_{t_{k}}, m\right)=\emptyset$, meaning that we improved the lower bound for $U C P(A)$ by 1 taking as a lower bound the submatrix $A^{\prime}+A_{i_{1}}+. .+A_{i_{k}}$. If $\operatorname{Sol}\left(A^{\prime}+A_{i_{1}}+. .+A_{i_{k}}, r\right)=\emptyset, r \geq m$ we improved the lower bound by $r-m+1$.

So an attractive idea is to start from a submatrix $A^{\prime}$ which is an $M S I R$ (since the solutions of an $M S I R$ can be represented compactly) and then to add rows to the $M S I R$ with the goal to improve the initial lower bound given by $|M S I R|$. The proposal relies on the intuition that, knowing $\operatorname{Sol}\left(A^{\prime}, m\right)$, it is not difficult to recalculate $\operatorname{Sol}\left(A^{\prime}+A_{p}, m\right)$ and, adding one row at a time, eventually we may reach the desired lower bound improvement. In
Section 4.1 we discuss how to recalculate solutions. This "naive" way of raising the lower bound may require too much memory. In Sections 4.2 and 5 we introduce a technique to avoid the problem: clustering solutions in cubes and branching by clusters.

To motivate the theory that will be developed, we show by an example how to raise the lower bound incrementally. Consider the following matrix $A_{N}$ that cannot be reduced by dominance.

\begin{tabular}{l|cccccccccc} 
& 0 & 1 & 2 & 3 & 4 & 5 & 6 & 7 & 8 & 9 \\
\hline 0 & 1 & 1 &. &. &. &. &. &. &. &. \\
1 &. &. & 1 & 1 &. &. &. &. &. &. \\
2 &. &. &. &. & 1 & 1 &. &. &. &. \\
3 &. &. &. &. &. &. & 1 & 1 &. &. \\
4 & 1 &. &. &. &. &. &. &. & 1 &. \\
5 &. & 1 &. &. &. &. &. &. &. &. \\
6 &. &. & 1 &. &. &. &. &. & 1 &. \\
7 &. &. &. & 1 &. &. &. &. &. & 1 \\
8 &. &. &. &. &. & 1 & 1 &. &. & 1 \\
9 &. &. &. &. & 1 &. &. & 1 &. &. \\
\end{tabular}

Suppose that $A_{N}$ is the submatrix corresponding to the node $N$ of a column branching search tree, such that the cost of the best solution found is 7 and the partial solution $S o l$ contains 1 column.

An $M S I R$ is made by the 4 rows $A_{0}, A_{1}, A_{2}$ and $A_{3}$. Since $\operatorname{cost}($ best $)-\operatorname{cost}(S o l)-|M S I R|=2$, potentially the lower bound could be raised by 2 . The set of irredundant solutions of $U C P(M S I R)$ is equal to $C_{0}=\{0,1\} \times\{2,3\} \times\{4,5\} \times\{6,7\}$. Select row $A_{4}$ from the rest of the matrix. The solutions of the matrix made by the rows $A_{0}, A_{1}, A_{2}, A_{3}$ and $A_{4}$ are represented by the union of the following two sets: $C_{1}=\{0\} \times\{2,3\} \times\{4,5\} \times\{6,7\}$ and $C_{2}=\{1\} \times\{2,3\} \times\{4,5\} \times\{6,7\} \times\{8\}$.

Consider independently the sets $C_{1}$ and $C_{2}$ starting with the solutions from $C_{1}$. Select row $A_{5}$ that is not covered by any solution in $C_{1}$. So each solution from $C_{1}$ must be augmented by a column covering $A_{5}$, which transforms $C_{1}$ into $C_{1}^{\prime}=\{0\} \times\{2,3\} \times$ $\{4,5\} \times\{6,7\} \times\{1,8\}$. Note that all solutions in $C_{1}^{\prime}$ consist of 5 columns. Now select row $A_{6}$. The solutions that have 5 columns, cover $A_{6}$, and can be obtained from the solutions in $C_{1}^{\prime}$ form the set $C_{1}^{\prime \prime}=\{0\} \times\{2\} \times\{4,5\} \times\{6,7\} \times\{1,8\}$. But no solution in $C_{1}^{\prime \prime}$ covers row $A_{7}$ and therefore each solution from the set must be augmented by a column to cover also $A_{7}$. It means that no solution from $C_{1}$ can be "extended" to a set of $\leq 5$ columns covering all rows from $A_{N}$.

Consider solutions from $C_{2}$. If we select row $A_{6}$, then all the solutions that have 5 columns, cover $A_{6}$ and can be obtained from the solutions in $C_{2}$ form the set $C_{2}^{\prime}=\{1\} \times\{2\} \times\{4,5\} \times\{6,7\} \times\{8\}$. But no solution in $C_{2}^{\prime}$ covers row $A_{7}$ and therefore each solution from the set must be augmented by a column to cover also $A_{7}$. It means that no solution from $C_{2}$ can be "extended" to a set of $\leq 5$ columns covering all rows from $A_{N}$. So the lower bound is raised to 6 and the branch corresponding to $A_{N}$ can be pruned.

\section{Representation and Recomputation of the Solu- tions}

In order to present the algorithm for raising the lower bound we must describe how the set of solutions of a matrix is represented and updated.

\subsection{Recomputation of the Solutions}

Let $A^{\prime}$ be a submatrix of $A$ and $A_{p}$ a row from $\operatorname{Row}(A) \backslash \operatorname{Row}\left(A^{\prime}\right)$. Let $S$ be a solution of $U C P(A)$. Denote by $O\left(A_{p}\right)$ the set $\left\{j \mid A_{p j}=1\right\}$, i.e., the set of all columns covering $A_{p}$ and by 
$\operatorname{Rec}\left(A^{\prime}+A_{p}, S\right)$ the set of solutions of $U C P\left(A^{\prime}+A_{p}\right)$ obtained according to the following rules:

1. if $S$ is a solution of $U C P\left(A^{\prime}+A_{p}\right)$, then $\operatorname{Rec}\left(A^{\prime}+A_{p}, S\right)=$ $\{S\}$;

2. if $S$ is not a solution of $U C P\left(A^{\prime}+A_{p}\right)$, i.e., no column of $S$ covers $A_{p}$ then $\operatorname{Rec}\left(A^{\prime}+A_{p}, S\right)=\left\{S \cup\{j\} \mid j \in O\left(A_{p}\right)\right\}$.

So $\operatorname{Rec}\left(A^{\prime}+A_{p}, S\right)$ gives the solutions of $U C P\left(A^{\prime}+A_{p}\right)$ that can be obtained from the solution $S$ of $U C P\left(A^{\prime}\right)$. According to 2 ., if $S$ is not a solution of $U C P\left(A^{\prime}+A_{p}\right)$, then we obtain $\left|O\left(A_{p}\right)\right|$ solutions of $U C P\left(A^{\prime}+A_{p}\right)$ by adding to $S$ the columns covering $A_{p}$.

Theorem 4.1 For any irredundant solution $S^{*} \in U C P\left(A^{\prime}+A_{p}\right)$ there is an irredundant solution $S \in U C P\left(A^{\prime}\right)$ such that $S^{*}$ is an element of $\operatorname{Rec}\left(A^{\prime}+A_{p}, S\right)$.

The proof of the theorem is omitted for lack of space. There are examples showing that $\operatorname{Rec}\left(A^{\prime}+A_{p}, S\right)$ may contain also redundant solutions.

Corollary 4.1 Let Sol be a set containing all irredundant solutions of $U C P\left(A^{\prime}\right)$. Let Sol $=\bigcup_{S \in S o l} \operatorname{Rec}\left(A^{\prime}+A p, S\right)$, then Sol* contains every irredundant solution $S^{*} \in U C P\left(A^{\prime}+A p\right)$.

Proof. It is a direct consequence of Theorem 4.1. $\square$

\subsection{Cubes of Solutions}

In line of principle, given the operator $R e c$, one could add one row at a time to $A^{\prime}$ and build the set of irredundant solutions of $U C P(A)$ from the set of irredundant solutions of $U C P\left(A^{\prime}\right)$. This "naive" approach must be discarded because of two disadvantages:

1. The size of the set of irredundant solutions may grow exponentially in the number of added rows.

2. Suppose that we want to raise the lower bound of $M S I R$ by small $n$ and that $S$ is a solution of $U C P(M S I R)$. It may happen that in order to raise $S$ by $n$ we need to add only a small set of rows from $\operatorname{Row}(A) \backslash \operatorname{Row}(M S I R)$. Denote the set $R(S)$. Let $S^{\prime}$ be another solution of $U C P(M S I R)$ and suppose that to raise it by $n$ we need to add a small set of rows $R\left(S^{\prime}\right)$. The problem is that $R(S)$ and $R\left(S^{\prime}\right)$ are usually different. In other words, when we add rows to $M S I R$ we want to add a minimal number of rows which raise all solutions of $M S I R$ by $n$. But, since these small sets $R(S)$ are usually different for different solutions $S$ from $U C P(M S I R)$, we actually need to add almost all rows.

To solve the previous issues we propose to clusterize solutions that can be raised by the same rows from $\operatorname{Row}(A) \backslash \operatorname{Row}(M S I R)$. This is achieved by the introduction of cubes of solutions, a data structure inspired by multi-valued cubes. Applying the operator Rec to a cube of solutions one obtains a collection of cubes of solutions, thereby providing a clusterization of the recomputed solutions. This will support later the design of a raising algorithm based on branching in clusters of solutions, each cluster being one of the recomputed cubes of solutions.

Note however that cubes should not be considered as the only convenient way to clusterize solutions. We believe that studying clusterizations based on different data structures, e.g., binary decision diagrams, will yield interesting results.

As anticipated, we represent the solutions of $U C P(A)$ by sets with a structure of multi-valued cubes [8]. We define a cube to be the set $C=D_{1} \times \cdots \times D_{d}$ where $D_{i} \cap D_{j}=\emptyset, i \neq j$ and $D_{i} \subset \operatorname{Col}(A)$, $1 \leq i, j \leq d$. The subsets $D_{i}$ are the domains of cube $C$. So cube $C$ denotes a set of sets consisting of $d$ columns. In contrast to standard cubes used for the representation of multi-valued functions here cubes may have different numbers of domains. For example, if $|\operatorname{Col}(A)|=10$, then sets $C_{1}=\{1,5\} \times\{2,6,7\} \times\{3,4\}$ and $C_{2}=\{1\} \times\{2,4\} \times\{3,7\} \times\{5,6,10\}$ are both cubes.

Let $A^{\prime}$ be a $M S I R$ of $A$. The set of all irredundant solutions (which are at the same time minimum) of $U C P\left(A^{\prime}\right)$ can be represented as the cube $O\left(A_{i_{1}}\right) \times \cdots \times O\left(A_{i_{d}}\right)$, where $A_{i_{1}}, \cdots, A_{i_{d}}$ are the rows forming $A^{\prime}$.

Let $A^{\prime}$ be a submatrix of $A$ and $A_{p}$ be a row from $\operatorname{Row}(A) \backslash$ Row $\left(A^{\prime}\right)$. Let $C=D_{1} \times \cdots \times D_{d}$ be a cube of solutions of $U C P\left(A^{\prime}\right)$. From the definition of the Rec operator it follows that

$$
\operatorname{Rec}\left(A^{\prime}+A_{p}, C\right)=\operatorname{part1}(C) \cup \operatorname{part} 2(C) \times O\left(A_{p}\right)
$$

where part $1(C)$ is the set of solutions contained in $C$ which cover $A_{p}$ and $\operatorname{part2}(C)$ is the set of solutions contained in $C$ which do not cover $A_{p}$.

There are three cases:

1. If $D_{i} \subseteq O\left(A_{p}\right)$ for some $i, 1 \leq i \leq d$, then any solution from $C$ covers the row $A_{p}$ and so $\operatorname{Rec}\left(A^{\prime}+A_{p}, C\right)=C$.

2. If $O\left(A_{p}\right) \cap D_{i}=\emptyset$ for any $i, 1 \leq i \leq d$, then no solution from $C$ covers $A_{p}$ and so $\operatorname{Rec}\left(A^{\prime}+A_{p}, C\right)=C \times O\left(A_{p}\right)=$ $D_{1} \times \cdots \times D_{d} \times O\left(A_{p}\right)$.

3. If 1. and 2. are not true, i.e., no $D_{i}$ is a subset of $O\left(A_{p}\right)$ and $O\left(A_{p}\right)$ intersects at least one domain (without loss of generality, we assume that $A_{p}$ intersects the first $r$ domains, i.e., $D_{1}, \cdots, D_{r}$ ), then cube $C$ can be partitioned into the following $r+1$ pairwise not intersecting cubes:

$$
\begin{aligned}
C_{1}= & D_{1} \cap O\left(A_{p}\right) \times D_{2} \times \cdots \times D_{d} \\
C_{2}= & D_{1} \backslash O\left(A_{p}\right) \times D_{2} \cap O\left(A_{p}\right) \times D_{3} \times \cdots \times D_{d} \\
C_{3}= & D_{1} \backslash O\left(A_{p}\right) \times D_{2} \backslash O\left(A_{p}\right) \times D_{3} \cap O\left(A_{p}\right) \times \\
& \times D_{4} \times \cdots \times D_{d} \\
& \cdots \\
C_{r}= & D_{1} \backslash O\left(A_{p}\right) \times \cdots \times D_{r-1} \backslash O\left(A_{p}\right) \times \\
& \times D_{r} \cap O\left(A_{p}\right) \times D_{r+1} \times \cdots \times D_{d}
\end{aligned}
$$

It is not hard to check that the union $C_{1} \cup \cdots \cup C_{r+1}$ gives the cube $C$ and that for any pair $C_{i}, C_{j}, i \neq j, C_{i} \cap C_{j}=\emptyset$. Moreover, the first $r$ cubes give the solutions of $U C P\left(A^{\prime}\right)$ from $C$ which cover $A_{p}$ and the cube $C_{r+1}$ gives the solutions of $U C P\left(A^{\prime}\right)$ from $C$ which do not cover $A_{p}$. Therefore

$$
\operatorname{part1}(C)=C_{1} \cup \cdots \cup C_{r}, \quad \operatorname{part2}(C)=C_{r+1} .
$$

Equations 1-3 realize the Rec operator as defined in Section 4.1 and characterized by Theorem 4.1. Notice that here we force the $R e c$ operator to generate non-intersecting cubes of solutions; this is not a consequence of the definition of Rec, but is an additional requirement introduced now to avoid considering the same partial solution in more than one branch.

We mentioned that in the computation of Rec some redundant solutions may be introduced. The following revised definition of $R e c$ avoids the generation of obviously redundant solutions obtained from the application of formula (1). Namely, any solution $S^{\prime}$ of $U C P\left(A^{\prime}+A_{p}\right)$ from part $2(C) \times O\left(A_{p}\right)$ that strictly contains a solution $S^{\prime \prime}$ of $U C P\left(A^{\prime}+A_{p}\right)$ from part $1(C)$ is redundant since it contains more columns than $S^{\prime \prime}$. 
Theorem 4.2 If the computation of the Rec operator is modified as follows:

$$
\begin{aligned}
\operatorname{Rec}\left(A^{\prime}+A_{p}, C\right)= & \operatorname{part1}(C) \cup \operatorname{part} 2(C) \times \\
& \times\left[O\left(A_{p}\right) \backslash\left(D_{1} \cup \cdots \cup D_{d}\right)\right]
\end{aligned}
$$

no irredundant solution of $A^{\prime}+A_{p}$ is discarded.

The proof of the theorem is omitted for lack of space.

\section{The Raising Procedure}

\subsection{Overview of the Raising Algorithm}

As anticipated in Section 2, we propose an $n$-raiser procedure which is called in the column branching mode as described in Fig. 1. Let $A$ be the covering matrix corresponding to the node where $n$-raiser is invoked and $A^{\prime}$ be an $M S I R$ of $A$. We start with the set of irredundant solutions of $U C P\left(A^{\prime}\right)$, represented by the cube $C=O\left(A_{i_{1}}\right) \times \cdots \times O\left(A_{i_{d}}\right)$, in which $A_{i_{1}}, \cdots, A_{i_{d}}$ are the rows in the $M S I R$. Then choose a "good" row of $A$ from those not in $A^{\prime}$, say row $A_{p}$. According to Equations (1-5), $\operatorname{Rec}\left(M S I R(A)+A_{p}, C\right)$ can be represented by $r+1$ cubes where $r$ is the number of rows of the $M S I R(A)$ intersecting $A_{p}$. Then perform recursively the process for each of the $r+1$ cubes, i.e., choose a new row from those not yet selected for each of the $r+1$ cubes of solutions and split each cube according to Equations (1-5).

The process can be described by a search tree, called cube branching tree. The initial cube of solutions $C$ corresponds to the root node, to which we associate also a pair of matrices $M S I R(A)$ and $A-M S I R(A)$ (i.e., matrix $A$ without the rows of $M S I R(A)$ ). In each node a choice of an unselected row from the second matrix of the node is made. The chosen row is removed from the second matrix of the pair and added to the first matrix of the pair. So the first matrix gives a "lower bound submatrix" for the node.

The number of branches leaving a node is equal to the number of cubes in which the cube corresponding to the node is partitioned by the Rec operation, and each child of a node gets one of the cubes obtained after splitting. So the cube corresponding to a node represents a set of solutions covering the first submatrix of the pair.

The flow of $n$-raiser is shown in Fig. 2 . The recursion terminates if one of the two following conditions hold:

1. There is a node such that there are no rows left in the second matrix of the pair and the corresponding cube has $k$ domains, where $k<|M S I R|+n$. This means that the lower bound $|M S I R|$ cannot be improved by $n$ and any solution from $C u b e$ consists of fewer columns than the current best one, since $n$-raiser is invoked if $|M S I R|+n+\operatorname{cost}(S o l)=$ cost(best) where $S o l$ is the partial solution found in the column branching mode before invoking the raiser. Then a solution from $C u b e$ is selected as the current best and the range of raiser is reduced to $n-(\operatorname{cost}($ old best $)-\operatorname{cost}($ best $))$ since the gap between the current best solution and MSIR is reduced.

2. From all branches, nodes are reached corresponding to cubes with a number of domains greater than $|M S I R|+n$. In this case the lower bound has been raised to $|M S I R|+n$, since no solution $S$ of $U C P(A)$ exists such that $|S| \leq|M S I R|+n$.

If neither pruning condition holds the procedure raise_or srim is invoked to address the following two cases, which let us modify the cube of solutions without branching:

1. If a row $A_{p}$ exists such that no solution from $C u b e$ covers $A_{p}$, then there is no splitting of the cube, since Rec yields only one cube $C \times O\left(A_{p}\right)$. Row $A_{p}$ is removed from $A^{\prime \prime}$ and added to $A^{\prime}$.
2. If there exists a row $A_{p}$ intersecting only one domain $D_{i}$ of $C u b e$ and the number of domains in $C u b e$ is equal to $|M S I R|+n-1$ then only two cubes are generated after splitting. The first cube is from part $1(C u b e)$ and is obtained by reducing domain $D_{i}$ to $D_{i} \cap O\left(A_{p}\right)$. The second cube has one more domain and can be discarded since the total number of domains in the cube is $|M S I R|+n$. Row $A_{p}$ is removed from $A^{\prime \prime}$ and added to $A^{\prime}$.

The previous conditions are checked in raise_or_trim by iterating through the rows of $A^{\prime \prime}$ until both conditions are false for any row from $A^{\prime \prime}$.

After all these special cases have been addressed, a new row $A_{p}$ is selected by select row. The row $A_{p}$ is removed from $A$ and drives the splitting of $C u b e$. The strategy to select the best row in order to split the current $C$ ube, before calling recursively raiser, looks for the row of $A$ which intersects the minimum number of domains of $C$ ube. The reason is to reduce the number of branches from the node, i.e., the number of domains intersecting the row to be added plus 1 . In case of ties between different rows, the row having the highest weight is chosen. The weight of a row $A_{p}$ is defined as:

$$
\prod_{k=1}^{m} \frac{\left|D_{i_{k}}^{\prime}\right|}{\left|D_{i_{k}}\right|}
$$

where $m$ is the number of domains of $C u b e$ intersecting $A_{p}, D_{i_{k}}$ is a domain intersected by $A_{p}$ and $D_{i_{k}}^{\prime}=D_{i_{k}} \backslash O\left(A_{p}\right)$. So the weight of $A_{p}$ is just the fraction of solutions from $C u b e$ that do not cover $A_{p}$, which we want to maximize when selecting a new row. If $D_{i_{k}}^{\prime}=\emptyset$, for some $k$, this means that $A_{p}$ is covered by any solution from $C$ ube. Such a row is simply removed from $A^{\prime \prime}$ and added to $A^{\prime}$.

\subsection{Correctness of n-raiser}

The correctness of the $n$-raiser procedure, applied to matrix $A$ with lower bound $|M S I R(A)|$, can be argued using the notions of subsolution or partial solution and of complete set of solutions, introduced as follows.

A set $S^{\prime}$ of columns of $A$ is a subsolution or partial solution of $U C P(A)$ if it is a solution of a subproblem $A^{\prime}$, but is not a solution of $U C P(A)$.

Let $C$ be the cube of subsolutions corresponding to $M S I R(A)$, then $C$ has the property that for any solution $S$ of $U C P(A)$ there is a subsolution from $C$ which is contained in $S$. Indeed, since $S$ covers all the rows of $A$, including those contained in $M S I R(A)$, then $S$ contains $|M S I R(A)|$ columns covering the submatrix $M S I R(A)$ that form a subsolution from $C$. A set of subsolutions is complete if for any solution $S$ of $U C P(A)$ there is a subsolution from the set which is contained in $S$. So the set of subsolutions contained in the cube $C$ is complete.

Let $S^{\prime}$ be a solution of subproblem $U C P\left(A^{\prime}\right)$. Denote by $\operatorname{Gen}\left(S^{\prime}\right)$ the set of irredundant solutions of $U C P(A)$ that contain $S^{\prime}$. Similarly, if $C$ is a set of partial solutions, denote by $\operatorname{Gen}(C)$ the set of irredundant solutions of $U C P(A)$, each of which contains a solution from $C$.

Lemma 5.1 Let $S^{\prime}$ be a solution of $U C P\left(A^{\prime}\right)$ and $A_{p}$ be a row from $\operatorname{Row}(A) \backslash \operatorname{Row}\left(A^{\prime}\right)$. Then $\operatorname{Gen}\left(S^{\prime}\right) \subseteq \operatorname{Gen}\left(\operatorname{Rec}\left(A^{\prime}+A_{p}, S^{\prime}\right)\right)$ where Rec is the recalculation operation defined in Section 4.1.

Proof. Let $S$ be a solution of $U C P(A)$ containing $S^{\prime}$, i.e., $S \in \operatorname{Gen}\left(S^{\prime}\right)$. If $S^{\prime}$ covers row $A_{p}$ then $\operatorname{Rec}\left(A^{\prime}+A_{p}, S^{\prime}\right)$ is equal to $\left\{S^{\prime}\right\}$ and so $\operatorname{Gen}\left(\operatorname{Rec}\left(A^{\prime}+A_{p}, S^{\prime}\right)\right)$ contains $S$. If $S^{\prime}$ does not cover $A_{p}$, then $\operatorname{Rec}\left(A^{\prime}+A_{p}, S^{\prime}\right)$ contains every solution $S^{\prime} \cup\{j\}, j \in O\left(A_{p}\right)$. Moreover, $S$ contains $S^{\prime}$ and, since it covers $A_{p}$, it obviously contains a column $j \in O\left(A_{p}\right)$. So again 
${ }^{*}$ n-raiser returns an empty solution if lower bound of $U C P(A)$ can be raised to $|M S I R|+n$. If not, it returns a current minimum solution of $U C P(A) * 1$

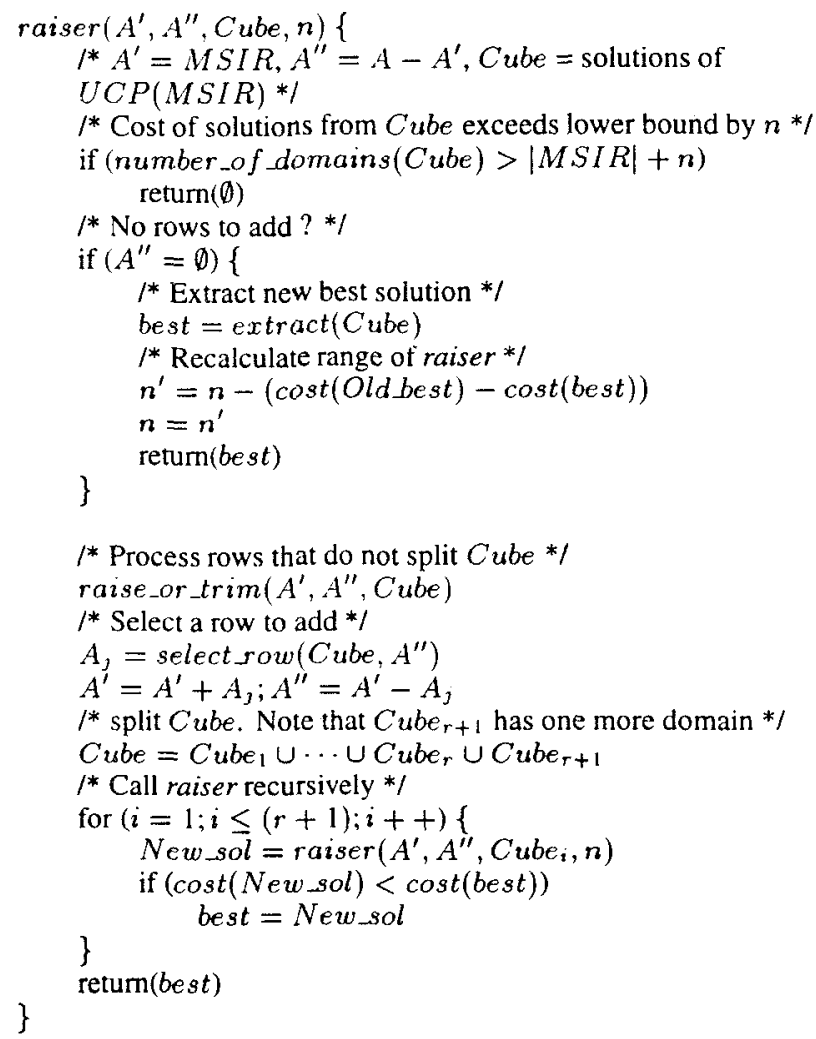

Figure 2: n-raiser algorithm

$\operatorname{Gen}\left(\operatorname{Rec}\left(A^{\prime}+A_{p}, S^{\prime}\right)\right)$ contains $S$.

From Lemma 5.1 it follows that the Rec operation preserves the completness of a set of subsolutions.

Theorem 5.1 The n-raiser procedure finds correctly a largerlower bound or a smaller upper bound.

Proof. $n$-raiser starts with the set of solutions of $U C P(M S I R)$, which is a complete set of partial solutions of $U C P(A)$. Since the Rec operation preserves completness, the set of all "boundary" cubes, i.e., cubes corresponding to either leaf nodes of the search tree or to the nodes not yet split, is a complete set of partial solutions. When we apply an $n$-raiser to $A$ we actually try to find a complete set of partial solutions containing at least $|M S I R(A)|+n$ columns. If such a set is found then no solution of $U C P(A)$ has less than $|M S I R(A)|+n$ columns, and so the procedure $n$-raiser succeeds in increasing the lower bound by $n$.

Suppose that there is no complete set of partial solutions consisting of at least $|M S I R(A)|+n$ columns. It means that $n$-raiser finds a leaf node with a cube containing solutions of $|M S I R(A)|+n^{\prime}$ columns where $n^{\prime}<n$. In that case we update the $n$-raiser into an $n^{\prime}$-raiser and continue the search. If the $n^{\prime}$-raiser succeeds we return a solution of $|M S I R(A)|+n^{\prime}$ columns which is minimal.

If the $n^{\prime}$-raiser fails then there is a solution of $U C P(A)$ consisting of $|M S I R(A)|+n^{\prime \prime}$ columns, where $n^{\prime \prime}<n^{\prime}$. Then we update the $n^{\prime}$-raiser into an $n^{\prime \prime}$-raiser and continue the search. $\square$

\section{Experimental Results}

We have implemented a program AURA to solve UCP and we have compared it with the routine mincov available in ESPRESSO, with MINCOV $\perp L B$, that is our implementation of some features of SCHERZO and with the results of the real SCHERZO implemented by O. Coudert. The program SCHERzo is the most effective solver of UCP currently reported. Its main features have been described in the literature $[3,2,1]$; they include a better heuristic selection of the $M S I R$, logarithmic lower bound, left hand side lower bound, limit lower bound, and partition-based pruning. Of these features we have implemented in MINCOV LLB, to the best of our understanding of the original description, the following two: better heuristic selection of the MSIR and limit lower bound. The limit lower bound is a major novelty of SCHERZO, which accounts for strong savings in the number of nodes of the computation tree compared to the original mincov of ESPRESSO.

The benchmarks belong to three different classes: in Table there are difficult cases from the collection of ESPRESSO (we start from the matrix obtained by ESPRESSO after removing the essential primes), in Table 2 there are random generated matrices with varying row/column ratios and densities, in Table 3 there are matrices encoding constraints satisfaction problems from [9]. The experiments have been performed with a $2 \mathrm{~GB} 300 \mathrm{Mhz}$ Alpha with timeout set to 3 days of cputime.

The tables report two types of data for comparison: the number of nodes of the column branching computation tree and the running time. About the number of nodes we clarify that

1. AURA has two types of nodes: those of the column branching computation tree and those of the cube branching computation tree (called A-nodes in the tables). Indeed AURA follows a dual strategy, i.e., it builds the column branching computation tree, but when at a node the difference between the upper bound and the lower bound is less or equal to the raising parameter $r$ (or max Raiser), AURA calls the procedure raiser which builds a cube branching computation tree, appended at the node where raiser was called. So we need to report both numbers of nodes to measure a run of AURA.

2. Nodes of the cube branching computation tree usually take much less computing time than those of the column branching computation tree, even though it is not known a-priori a time ratio between the two types of nodes. The reason is that in each node of the column branching mode expensive procedures for finding dominance relations and the $M S I R$ are applied.

3. The raising parameter is an input to AURA. Currently we have experimented with some values and we report in the tables the value used in a specific run. The higher is the raising parameter, the fewer column branching nodes compared to cube branching nodes there will be. With a value high enough, there will be a single column node and the rest will be all row nodes.

We compared also with the real SCHERZO, whose author was kind enough to run for us the examples. There is a large gap in many cases between the results of SCHERZO and those of MINCOV LLB, which is our implementation of a subset of SCHERZo, A major reason may be that our reimplementation of the better heuristic selection of the $M S I R$, even though it follows the hint given by Coudert, in practice does not mimic well enough the one in SCHERZo; moreover, as already said, SCHERZO features additional improvements that we did not implement. It is important to underline that: 
1. both AURA and MINCOV LLB exploit the same re-implementation of Coudert's better heuristic selection of the MSIR:

2. AURA could be improved noticeably by reproducing more successfully the better heuristic selection of the MSIR or any other feature of SCHERZO. In other words, AURA demonstrates a dual search technique, which may benefit from other improvements to standard branch and bound.

3. in overall SCHERzo has been implemented more efficiently, as magnified also by the circumstance that it is comparatively faster on a slower machine.

The experiments show that AURA outperforms ESPRESSO and MINCOV $\perp$ LB. It is always faster and in the most difficult examples either it has a running time advantage up to two orders of magnitude or the other programs fail due to timeout ( 3 days) or spaceout $(2 G)$. Instead SCHERZO is a very tough competitor, which is faster on the examples from Table 1, but has a less effective pruning strategy in those of Tables 2 and 3, partially compensated by a better $M S I R$. The example sauciert $t$ is an extreme case where the virtues of AURA prevail. Recently $O$. Coudert kindly provided us with a copy of SCHERZO, to let us analyze in depth the comparative features of the two programs. We will report on the study as soon as done. We expect to transfer to AURA the better computation of the $M S I R$ apparently implemented in SCHERZO.

We do not have a systematic comparison with the results by $\mathrm{BCU}$, a recent ILP-based covering solver [5]. However, the intuition is that an algorithm based on linear programming is better suited for problems with a solution space diversified in the costs, i.e., for problems which are "closer" to numerical ones. To test the conjecture we asked the authors of [5] to run BCU on sauciert, whose solution space is poorly diversified (a minimum solution has 6 columns, while most of the irredundant solutions cost in the range from 6 to 8). BCU ran out of memory after 20000 seconds of computations (the information was kindly provided by S.Liao), while AURA completed the example in less than 3 minutes.

\section{Conclusions}

We have introduced a new technique to solve exactly a discrete optimization problem, based on the paradigm of "negative" thinking. The motivation is that when searching the space of solutions often a good solution is reached quickly and then it is improved only a few times before the optimum is found; so most of the solution space is explored to certify optimality, but it does not yield any improvement in the cost function. This suggests that more powerful lower bounding would speed up the search dramatically, as shown by the introduction of the limit lower bound [3]. Our approach is more radical because when we are dealing with a subspace of solutions unlikely to improve the upper bound, we switch the search strategy to a different one geared to raise the lower bound. To design a search strategy which realizes negative thinking we introduced cubes of solutions, a data structure inspired by multi-valued cubes. Applying the operator $R e c$ to a cube of solutions one obtains a collection of cubes of solution, thereby providing a natural clustering of the recomputed solutions. As argued in the paper, clustering is required to design a recursive algorithm based on branching in subsets of solutions and allows the lower bound to be raised independently starting from different subsets of solutions.

For illustration we applied our technique to the unate covering problem, usually solved exactly by a branch-and-bound procedure, where one lower bounds by means of an independent set of rows, and branches on columns. We have designed a dual search technique, called raiser, which is invoked when the difference between the upper bound and the lower bound is within a parameter max Raiser, that we are free to set. The procedure raiser tries to detect a hard core of the matrix to be solved (lower bound submatrix), augmenting an independent set of rows in order to increase incrementally the cardinality of the minimum solutions that cover it. Eventually either this incremental raising yields a lower bound that matches the current upper bound and so we are done, or we produce a better solution. Raiser defines a computation tree whose nodes have associated a lower bound submatrix and a cube of solutions. The selection of a next row induces the recomputation of all the solutions of the lower bound submatrix augmented by the next row, as disjoint cubes of solutions. Each such cube together with the augmented matrix defines a new node; operationally raiser calls itself recursively passing as parameters each such disjoint cube of solutions and the augmented lower bound submatrix. It would be interesting to explore a mixed approach where one accumulates some cubes of solutions at the same node and fewer recursive calls are made, trading off time vs. memory.

The reported experiments show that our program AURA, outperforms ESPRESSO and MINCOVLLB, which is the algorithm in ESPRESSO enhanced by our implementation of Coudert's limit lower bound. The package SCHERZO is faster than AURA on the examples from Table 1 , but it has a less effective pruning strategy in those of Tables 2 and 3, partially compensated by a better $M S I R$.

Future work includes a more careful study of some algorithmic design issues, like the selection of the next row, trading-off number of nodes vs. number of cubes stored in a node, and setting automatically and adaptively the raiser parameter.

A more basic line of research is the exploration of data structures different from cubes since the latter are just the simplest way of representing sets of partial solutions. We believe that studying various ways of representing implicitly sets of solutions is a promising direction of investigation to rescue branch-and-bound from its current limits. Another important direction of future research is to apply the negative thinking approach to other problems.

\section{References}

[1] O. Coudert. Two-level logic minimization: an overview. Integration, 17-2:97-140, October 1994.

[2] 0 . Coudert. On solving binate covering problems. In The Proceedings of the Design Automation Conference, pages 197202, June 1996.

[3] O. Coudert and J.C. Madre. New ideas for solving covering problems. In The Proceedings of the Design Automation Conference, pages 641-646, June 1995.

[4] T. Kam, T. Villa, R. Brayton, and A. Sangiovanni-Vincentelli. Synthesis of FSMs: functional optimization. Kluwer Academic Publishers, 1996.

[5] S. Liao and S. Devadas. Solving covering problems using LPR-based lower bounds. In The Proceedings of the Design Automation Conference, June 1997.

[6] J.-K. Rho and F. Somenzi. Stamina. Computer Program, 1991.

[7] R. Rudell. Espresso. Computer Program, 1987.

[8] R. Rudell and A. Sangiovanni-Vincentelli. Multiple-valued minimization for PLA optimization. IEEE Transactions on Computer-Aided Design, CAD-6:727-750, September 1987.

[9] Tiziano Villa. Encoding Problems in Logic Synthesis. PhD thesis, University of California, Berkeley, Electronics Research Laboratory, May 1995. Memorandum No. UCB/ERL M95/41. 


\begin{tabular}{|c|c|c|c|c|c|c|c|c|c|c|c|}
\hline \multirow[t]{2}{*}{ matrix } & \multirow[t]{2}{*}{$\overline{\mathrm{R}} \times \overline{\mathrm{C}(\text { Sparsity })}$} & \multirow[t]{2}{*}{ Sol. } & \multicolumn{2}{|c|}{ ESPRESSO } & \multicolumn{2}{|c|}{ SCHERZO } & \multicolumn{2}{|c|}{ MINCOV $\perp L B$} & \multicolumn{3}{|c|}{ AURA } \\
\hline & & & nodes & time & nodes & time & nodes & time & nodes/A-nodes & time & $\mathbf{r}$ \\
\hline exps & $680 \times 696(1.2 \%)$ & 76 & 13 & 0.0 & na & na & $\sqrt{3}$ & $\overline{0.0}$ & $13 / 0$ & 0.0 & 3 \\
\hline fout & $177 \times 431(2.4 \%)$ & 38 & 161 & 1.3 & na & na & 49 & 0.7 & $18 / 44$ & 0.2 & 2 \\
\hline $\max 512$ & $559 \times 515(1.3 \%)$ & 113 & 111 & 1.4 & na & na & 25 & 0.4 & $19 / 25$ & 0.4 & 3 \\
\hline addm 4 & $832 \times 1073(0.6 \%)$ & 165 & 121 & 3.6 & na & na & 29 & 1.1 & $17 / 11$ & 0.6 & 2 \\
\hline $\operatorname{mlp} 4$ & $530 \times 594(0.99 \%)$ & 109 & 2122 & 22.6 & 24 & 0.1 & 153 & 4.3 & $34 / 206$ & 1.3 & 3 \\
\hline pdc & $6904 \times 19021(0.34 \%)$ & 94 & 195 & 62.7 & 44 & 6.1 & 88 & 58 & $41 / 132$ & 52.9 & 3 \\
\hline lin.rom & $1030 \times 1076(0.9 \%)$ & 120 & 370 & 29.1 & 238 & 4.7 & 106 & 10.1 & $61 / 240$ & 7.7 & 3 \\
\hline ex 5 & $831 \times 2428(2 \%)$ & 37 & - & time & 616091 & 2450.5 & 597644 & 214300 & $155 / 169245$ & 1315.2 & 4 \\
\hline prom2 & $1924 \times 2611(0.31 \%)$ & 278 & - & time & 25993 & 5149.2 & - & time & $1478 / 1097624$ & 24071.4 & 3 \\
\hline $\max 1024$ & $1090 \times 1264(0.52 \%)$ & 245 & - & time & 531618 & 9583.6 & - & time & $12402 / 3850628$ & 36240 & 3 \\
\hline
\end{tabular}

Table 1: Results from Espresso Benchmarks

\begin{tabular}{|c|c|c|c|c|c|c|c|c|c|c|c|}
\hline \multirow[t]{2}{*}{ matrix } & \multirow{2}{*}{$\mathrm{R} \times \mathrm{C}$ (Sparsity) } & \multirow[t]{2}{*}{ Sol. } & \multicolumn{2}{|c|}{ ESPRESSO } & \multicolumn{2}{|c|}{ SCHERZO } & \multicolumn{2}{|c|}{ MINCOV_LLB } & \multicolumn{3}{|c|}{ AURA } \\
\hline & & & nodes & time & nodes & time & nodes & time & nodes/A-nodes & time & $r$ \\
\hline tc. 90 & $50 \times 100(90 \%)$ & $\overline{2}$ & 135 & $\overline{2.6}$ & 2 & 0.0 & $\overline{3}$ & 0.3 & $3 / 1$ & 0.1 & $\overline{3}$ \\
\hline tc. 70 & $50 \times 100(70 \%)$ & 2 & 135 & 3.5 & 2 & 0.0 & 3 & 0.2 & $3 / 1$ & 0.1 & 3 \\
\hline tc. 50 & $50 \times 100(50 \%)$ & 3 & 2569 & 13.9 & 107 & 0.6 & 107 & 2.3 & $5 / 32$ & 0.1 & 3 \\
\hline tc. 30 & $50 \times 100(30 \%)$ & 4 & 12047 & 37.8 & 65 & 0.3 & 1061 & 7.1 & $11 / 203$ & 0.2 & 3 \\
\hline tc. 10 & $50 \times 99(10 \%)$ & 8 & 843 & 3.3 & 90 & 0.1 & 131 & 0.7 & $17 / 166$ & 0.1 & 3 \\
\hline tr. 10 & $100 \times 50(20 \%)$ & 8 & 12466 & 59.6 & 2077 & 4.1 & 2232 & 21.1 & $94 / 2529$ & 2.9 & 3 \\
\hline $\operatorname{tr} .20$ & $100 \times 50(40 \%)$ & 5 & 16905 & 49 & 1823 & 3.9 & 2193 & 19.2 & $31 / 951$ & 1.7 & 3 \\
\hline tr. 30 & $100 \times 50(60 \%)$ & 3 & 947 & 9.5 & 63 & 0.9 & 61 & 3.4 & $5 / 26$ & 0.3 & 3 \\
\hline tr. 40 & $100 \times 50(80 \%)$ & 2 & 73 & 4.3 & 2 & 0.0 & 3 & 0.6 & $3 / 1$ & 0.3 & 3 \\
\hline ts. 90 & $100 \times 100(90 \%)$ & 2 & 175 & 21.2 & 2 & 0.0 & 3 & 2.6 & $3 / 1$ & 1 & 3 \\
\hline ts. 70 & $100 \times 100(70 \%)$ & 3 & 5083 & 47.0 & 167 & 5.3 & 163 & 15.8 & $5 / 112$ & 0.7 & 3 \\
\hline ts. 50 & $100 \times 100(50 \%)$ & 4 & 66147 & 316.4 & 4011 & 20.2 & 3137 & 67.3 & $7 / 1030$ & 1.6 & 3 \\
\hline ts. 30 & $100 \times 100(30 \%)$ & 5 & 116307 & 792.8 & 1752 & 8.5 & 8997 & 139.6 & $35 / 1108$ & 2.5 & 3 \\
\hline ts. 10 & $100 \times 100(10 \%)$ & 12 & - & time & 95573 & 187.3 & 175255 & 1255.1 & $5043 / 201091$ & 129.3 & 3 \\
\hline
\end{tabular}

Table 2: Results from Random Generated Matrices

\begin{tabular}{|c|c|c|c|c|c|c|c|c|c|c|c|}
\hline \multirow[t]{2}{*}{ matrix } & \multirow[t]{2}{*}{$\mathrm{R} \times \mathrm{C}($ Sparsity $)$} & \multirow[t]{2}{*}{ Sol. } & \multicolumn{2}{|c|}{ ESPRESSO } & \multicolumn{2}{|c|}{ SCHERZO } & \multicolumn{2}{|c|}{ MINCOV_LB } & \multicolumn{3}{|c|}{$\overline{\text { AURA }}$} \\
\hline & & & nodes & time & nodes & time & nodes & time & nodes/A-nodes & time & r \\
\hline$\overline{\text { bbara.t }}$ & $45 \times 26(41 \%)$ & $\overline{7}$ & 61 & $0 . \overline{02}$ & $\overline{0}$ & 0.0 & 7 & 0 & $7 / 2$ & $\overline{0}$ & 3 \\
\hline $\mathrm{dk} 512 \mathrm{x} . \mathrm{t}$ & $91 \times 59(45 \%)$ & 6 & 213 & 0.24 & 55 & 0.0 & 57 & 0.15 & $9 / 24$ & 0.04 & 3 \\
\hline ex4inp.t & $91 \times 240(46 \%)$ & 5 & 5279 & 16.81 & 17 & 0.3 & 19 & 0.66 & $9 / 14$ & 0.27 & 3 \\
\hline ex5inp.t & $36 \times 34(48 \%)$ & 4 & 64 & 0.05 & 4 & 0.0 & 6 & 0.01 & $6 / 2$ & 0.01 & 3 \\
\hline ex6inp.t & $28 \times 96(48 \%)$ & 4 & 639 & 0.54 & 35 & 0.0 & 103 & 0.28 & $7 / 23$ & 0.03 & 3 \\
\hline maincont.t & $105 \times 67(35 \%)$ & 7 & 504 & 0.69 & 68 & 0.0 & 101 & 0.4 & $11 / 12$ & 0.06 & 3 \\
\hline ricks.t & $78 \times 363(47 \%)$ & 5 & 20 & 0.37 & 10 & 0.2 & 12 & 0.36 & $8 / 43$ & 0.33 & 3 \\
\hline saucier.t & $171 \times 6207(47 \%)$ & 6 & - & mem & 186927 & 5441.0 & & mem & $10 \cap 6$ & 222.47 & 3 \\
\hline
\end{tabular}

Table 3: Results from Encoding Problem Matrices 